\title{
THE INFLUENCE OF BINDING MATERIAL ON POROUS STRUCTURE OF SHAPED HOPCALITE
}

\author{
N.K. Kulikovª, S.G. Kireeva, A.O. Shevchenko ${ }^{a}$, V.M. Mukhin ${ }^{a}$, S.N. Tkachenko ${ }^{\text {b }}$ T.G. Lupascu ${ }^{\mathrm{c}}$ \\ JSC EHMZ, Elektrostal, Moscow region, 144001, K.Marks str., 1 \\ aJSC ENPO "Neorganika", Elektrostal, Moscow region, 144001, K.Marks str.,4 \\ ${ }^{b} \mathrm{MSU}$ by M.V. Lomonosov, Chemistry faculty, Moscow \\ 'Institute of Chemistry of Academy of Sciences Moldova
}

\begin{abstract}
The authors have investigated the equilibrated adsorption of water vapors on GFG hopcalite, which was obtained using the extrusion shaping method, with bentonite clay as the binding compound. In the frames of the BET model, the values of the monolayer capacity and the size of medium area occupied by the water molecule in the filled monolayer have been determined. The distribution of pores according to their sizes has been evaluated. It has been established that the modification of the bentonitic clay allows directed construction of the hopcalite porous structure, i.e. the formation of the mesoporous structure with a narrow distribution of the pores capacities by sizes, which was achieved varying the sizes of binding compound particles.
\end{abstract}

Keywords: bentonitic clay, hopcalite, adsorption, porosity, catalytic properties.

It is known that physical-chemical properties of manganese oxide catalysts (MOC) that are used for oxidation of carbon monoxide and ozone decomposition, greatly depend on the base phase structure - manganese dioxide and also on the method and conditions of the latter receiving [1,2]. Besides, catalyst properties may be also influenced by the binding material. For example, the use of different binding materials in shaped catalysts produced on the basis on $\mathrm{MnO}_{2} / \mathrm{CuO}$ determines different adsorption properties, in particular in connection with water vapors. So, in case of utilization of bentonitic clay (BC), talum and calcium oxide the value of monolayer capacity according to BET is correspondingly [3] 40, 38 and $24 \mathrm{mmole} / \mathrm{g}$.

During the present work we investigated the influence of properties of one and the same binding material on adsorption properties of hopcalite, consisting of $\mathrm{MnO}_{2} / \mathrm{CuO}$ and $10 \%$ by weight of $\mathrm{BC}$. The following samples were chosen as investigation objects: industrial hopcalite GFG as the a control and modified hopcalite GFG-M for a development type. The samples used as investigation objects differed in binding particles size: maximum distribution treatment was $12 \mu \mathrm{m}$ for GFG, $4 \mu \mathrm{m}$ for GFG-M.

Bentonitic clay (BC) used as binding material in hopcalite production has complicated chemical and mineralogical structure [4]. One of the main minerals is montmorillonite but also quartz, amorphous aluminum silicates, hydroxides and other compounds are present. Thus BC consists both of clayey components, providing binding properties, and clean components which are catalyst ballast. Clayey fraction separation was carried out in the following way: $\mathrm{BC}$ hinge was poured over with tenfold quantity of water and kept at room temperature with periodical mixing. After some astronomical days clayey fraction was separated from settled clean components. The final rate appeared to be $\sim 40 \%$, converted to dry substance. This was followed by the mixing of manganese dioxide and black copper oxide with modified binder in the ratio taken in active production. Subsequent operations were: forming of granules $1 \mathrm{~mm}$ in diameter by the extrusion method, drying, crushing, separation of principal fraction - cylindrical particles $2-5 \mathrm{~mm}$ in length and thermal treatment at operating practice characteristics similar to those taken in hopcalite industrial production.

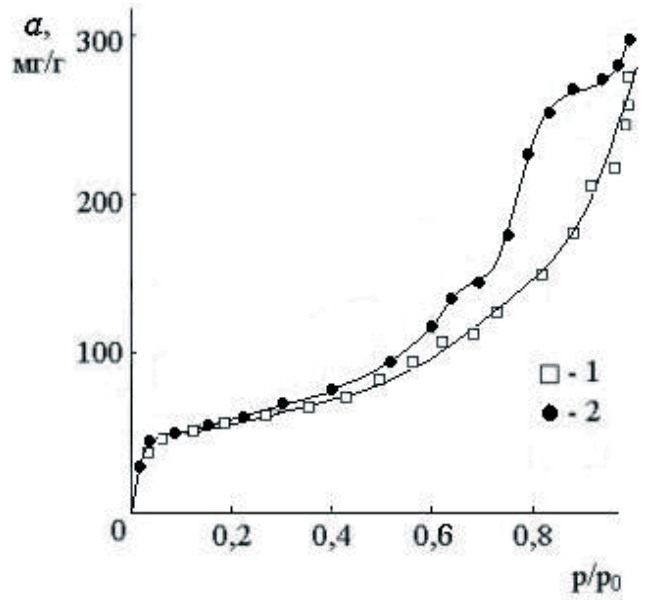

Fig.1 Water vapors adsorption on GFG (1) and GFG-M (2) hopcalites. 
Adsorption investigation method, equipment, way and conditions of sample training before the metrology were analogous to those described in the work [5].

Samples specific surface was defined by means of nitrogen thermal desorption. Figure 1 shows isotherms of water vapors adsorption on GFG and GFG-M hopcalites, measured at room temperature.

Both isotherms are described satisfactorily in the context of BET model [6] with the help of the calculation device, which determined constant $\mathrm{C}$, monolayer capacity $\left(\mathrm{a}_{\mathrm{m}}\right)$ and the size of medium area occupied by the water molecule in the filled monolayer $(\omega)$. All the values are given in table 1 .

Samples adsorption properties in the monolayer area and closest monolayer area, situated next to it, are practically equal, because the beginning parts of isotherms coincide, and volumes of constant $\mathrm{C}, \mathrm{a}_{\mathrm{m}}$, monolayer capacity and $\omega$ differ slightly. But at the increase of equilibrium pressure of adsorbate beginning with $\sim 0,4 \mathrm{p} / \mathrm{p}_{\mathrm{o}}$, the differences in adsorption behavior of GFG and GFG-M are evident. According to the classification of Brunauer, Deming and Teller isotherm of sample GFG may be ascribed to II type, however for sample GFG-M in the area of high relative pressures isotherm part is of the IV type with characteristic inflection [6] which gives the possibility to track the moment of mesopore infill.

Table 1

Hopcalite adsorption characteristics

\begin{tabular}{|c|c|c|c|c|c|c|c|c|}
\hline \multirow[b]{2}{*}{ Sample } & \multirow[b]{2}{*}{$\mathrm{C}$} & \multicolumn{2}{|c|}{$a_{m}$} & \multirow[t]{2}{*}{$\mathrm{S}_{\mathrm{sp}}, \mathrm{m}^{2} \backslash \mathrm{g}$} & \multirow[b]{2}{*}{$\omega, \AA^{2}$} & \multicolumn{3}{|c|}{$\mathrm{S}_{\mathrm{me}}, \mathrm{m}^{2} \backslash \mathrm{g}$, in the interval } \\
\hline & & $m g \backslash g$ & mmole $\backslash g$ & & & $3-5 \mathrm{~nm}$ & $5-7 \mathrm{~nm}$ & $7-16 \mathrm{~nm}$ \\
\hline GFG & 42 & 38.7 & 2.15 & 156 & 12.1 & 13.7 & 4.1 & 4.2 \\
\hline GFG-M & 45 & $40 / 4$ & 2.24 & 180 & 13.3 & 26.5 & 7.6 & 1.8 \\
\hline
\end{tabular}

Information about pore structure of the solid, for example, pore distribution according to size, may be also obtained from experimental isotherms. Adsorption branches of isotherms have been chosen for the calculation. First, according to the modern point of view, it is more valid in the thermodynamic respect. Second, in real adsorption systems there is always interrelation pore influence (unlike ideal model of separate independent pores) and distribution, measured on adsorption branch, gives more correct information about pore structure.

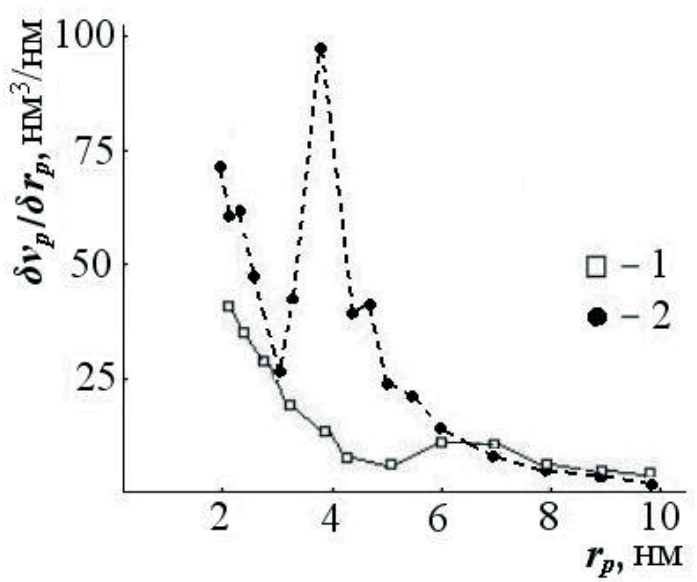

Figure 2. Distribution of pore volume according to sizes for GFG (1) and GFG-M (2).

On Roberts method [6] we carried out the measurement of pore distribution according sizes with the use of cylindrical pores model (fig. 2) and also of pore surface area in the range of 3-16 nm (table 1). To calculate adsorption film thickness we used Helsy equation [7]. The data given on fig. 2 and in the last three columns of table 1 are the evidence of great influence of binding material modification on GFG pore structure. If at 6-7 nm you can observe rather weak wave at distribution curve for initial hopcalite, then GFG-M at $3.8 \mathrm{~nm}$ is characterized by manifestly evident and rather intensive distribution maximum. Taking into account the form variety of making hopcalite particles and also the irregular nature of their arrangement, we can assume that the most likely reason of such effect is not only another binding material pore size but also the motive change of adsorbent gel-forming particles arrangement.

Actually, on one hand, the separation of clayey fraction from initial BC led to great decrease of binding material particle size as a result of swelling and aliquation. But on the other hand, for all that total quantity of binding material as in GFG, the use of modified BC in GFG-M provides 2.5 times more of clayey fraction which is just a binding component. This is confirmed by the fact that granules abrasion resistance is 77.8 and $85.2 \%$ for GFG and GFG-M correspondingly. Besides, BC modification led to certain compacting of catalyst granules: it is confirmed by bulk density growth, which is 986 and $1037 \mathrm{gldm}^{3}$ for GFG and GFG-M correspondingly. 
The specific surface of GFG-M sample is greater by $24 \mathrm{~m}^{2} \backslash \mathrm{g}$ than that of GFG. From the other hand, as the data of table 1 show, the difference between mesopores of mentioned samples is $13.9 \mathrm{~m}^{2} \backslash \mathrm{g}$. However taking into account the fact that there is a certain error at $\mathrm{S}_{\mathrm{sp}}$ determination and adsorption measurements, we have reasons to establish rather satisfactory coincidence of both calculation results and those received by the instrumental method. Hence, we can draw a conclusion about the correctness of application of chosen models for the description of equilibrium adsorption processes in the system "hopcalite - water". If initial hopcalite GFG is characterized with rather equal mesopore volume distribution according to their size, GFG-M is characterized with narrow distribution with maximum at $3.8 \mathrm{~nm}$.

Thus, bentonitic clay modification allows to construct hopcalite porous structure purposively, to be exact: to form mesoporous structure with narrow distribution of pore volume on sizes by means of variation of binding material particle size.

\section{Refferences}

[1] Golbraih Z.E. Manganese dioxide as an adsorbent // In the book: Active manganese dioxide.-L.:ONTI-Himteoret. 1937.-p.124-162.

[2] Rode E.Y. Manganese oxygen compounds.-M.: PH AN SSSR. 1953.-478 p..

[3] Kireev S.G., Mukhin V.M., Kireev A.S. and others. Theoretical problems of chemistry of surface, adsorption and chromatography: Thesis of rep. to X International conference. April, 24-28, 2006, Moscow-Klyazma.-M.: IFH RAN, 2006.-p.175.

[4] Keltsev N.V. Principles of adsorption technique. $2_{\text {nd }}$ edition, revised and complem.- M.:Chemistry. 1984.-592 p.

[5] Kireev S.G., Mukhin V.M., Tchebykin V.V. Modern state and development perspectives of adsorption theory: Coll. of mater. of IX International conference on theoretical questions of adsorption and adsorption chromatographyMoscow, 2001, April,24-26.- M.: IFH RAN. 2001.-p. 225-228.

[6] Greg S., Sing K. Adsorption, specific surface, porosity: Transl. from Engl. $2_{\text {nd }}$ edition-M.: Mir. 1984.-306 p.

[7] Komarov V.S. Adsorbents and their properties.-Minsk: Science and technique. 1977.-248 p. 